\title{
Community implementation of the 3 Wishes Project: an observational study of a compassionate end-of-life care initiative for critically ill patients
}

\author{
Brenda K. Reeve MD, Brittany B. Dennis MBBS PhD, William Dechert MSc, Barbara Longo RN BScN, \\ Diane Heels-Ansdell MSc, Alison Scholes, France J. Clarke RRT, John R.K. Arthur MDiv, \\ Deborah J. Cook MD MSc
}

\section{Abstract}

Background: The 3 Wishes Project (3WP) promotes a personalized dying experience by eliciting and facilitating individualized terminal wishes for patients, families and the clinicians caring for them. We aimed to evaluate the adaptability of the 3WP to a community intensive care unit (ICU), and to describe the patients cared for with this palliative approach, as well as local implementation strategies.

Methods: The 3WP was implemented in a 15-bed community hospital ICU in southern Ontario from 2017 to 2019 . In this observational, descriptive study, we invited adult patients ( $\geq 18 \mathrm{yr}$ ) whose risk of death was deemed to be $95 \%$ or greater by the attending physician, or patients undergoing withdrawal of life-support to participate. We abstracted patient data from medical records, as well as the type, timing and cost of each wish, which person or service made and facilitated each wish, and if and why wishes were completed or not. We summarized data both narratively and quantitatively.

Results: The 3WP helped to realize 479 (99.2\%) of 483 terminal wishes for 101 dying patients. This initiative was introduced as an interprofessional intervention and championed by nursing staff who were responsible for most patient enrolment and wish facilitation. Wishes included humanizing the ICU environment for the patient with belongings and blankets, musical performances, smudging and bathing ceremonies, and keepsakes. The cost was \$5.39 per patient (standard deviation \$22.40), with 430 (89.8\%) wishes incurring no cost. Wishes made directly by patients accounted for $30(6.2 \%)$ of wishes; those from family members and ICU staff accounted for $236(48.9 \%)$ and $238(49.3 \%)$ of wishes, respectively. The program comforted patients and their loved ones, motivating clinicians to sustain this end-of-life intervention.

Interpretation: We documented successful implementation of the 3WP in a community hospital, showing program adaptability and uptake outside of academic centres at relatively low cost. The lack of strict protocolization and personalized design of this intervention underscores its inherent flexibility, with potential to promote individualized end-of-life care in nonacademic hospital wards, homes or hospice.

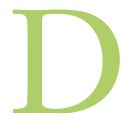

eath and dying are undoubtedly sacred processes, valued and experienced differently across cultures. In some Buddhist traditions, the last thought at the moment of death determines the character of the next reincarnation. For the First Nations Ojibway people, the time of death is a spiritual transcendence, where Mother Earth reclaims the physical form, and the Creator father carries the spirit to its origin place. ${ }^{1}$ Despite its sanctity, most deaths in Canada occur in hospital, ${ }^{2}$ guided by clinicians and often without an individualized approach.

In the intensive care unit (ICU), technology deployed for monitoring and treatment can render the setting impersonal, noisy and sterile for critically ill patients who are dying. Endof-life care is ideally congruent with the goals of the patient, sometimes expressed in written or verbal advanced directives, but often expressed by family members in real time during serious illness. Despite the high mortality rate of patients who are critically ill or injured, optimal strategies for providing personalized care to dying patients, and methods to help families navigate the dying and grieving processes, remain understudied in the ICU setting.

The 3 Wishes Project ( $3 W P$ ) was developed in an academic teaching hospital with the goal of bringing peace to a patient's final days and comforting families. This program

\section{Competing interests: None declared.}

This article has been peer reviewed.

Correspondence to: Deborah Cook, debcook@mcmaster.ca

CMAJ Open 2021. DOI:10.9778/cmajo.20200273 
aims to honour the individual, promote patient legacy, support families, and enrich relationships among patients, families and clinicians - all integral to patient- and familycentred end-of-life care ${ }^{3-5}$ - by eliciting and facilitating terminal wishes for dying patients, their loved ones and the clinicians caring for them. Wishes range from enhancing the clinical environment with personal belongings, to life celebrations, pet visitation, religious ceremonies and musical performances. ${ }^{4}$ Multicentred program evaluation in 4 North American academic centres showed that the $3 \mathrm{WP}$ is a valued, affordable and sustainable program that honours the inherent dignity of each patient. ${ }^{4}$

However, the transferability of this program to a community setting is uncertain and may be challenging, given a potentially less diverse multidisciplinary team of caregivers, fewer general house-staff to elicit and implement wishes and less available funding. In Canada, ICUs in community hospitals historically have not been as engaged in data collection for research and quality improvement, and many lack dedicated research staff.

The overall objective of this study was to evaluate the adaptability of the $3 \mathrm{WP}$ to a community hospital ICU, and to describe the patients cared for with this palliative approach and local program implementation strategies. By studying adaptability outside of academic centres, we assess whether this program can be successfully introduced and accepted, as judged by patient enrolment, staff participation and costs.

\section{Methods}

\section{Setting and design}

We implemented the 3WP between January 2017 and December 2019 in a level 3, 15-bed ICU at Brantford General Hospital, a community hospital in southern Ontario that is affiliated with McMaster University. The $3 \mathrm{WP}$ begins as a conversation that allows the bedside team to learn about the dying patient as a person, namely their interests, values and aspects of their life that are important to them..$^{3-5}$ The team helps to elicit and facilitate individualized wishes made by patients and their families, with additional acts of compassion from clinicians representing additional wishes. We evaluated the program using descriptive analysis.

\section{Participants}

Adult patients ( $\geq 18 \mathrm{yr}$ ) were eligible to participate if their risk of death in the ICU was deemed to be $95 \%$ or greater by the attending physician, or if withdrawal of life-sustaining technology was planned. Eligibility was determined via discussions with the most responsible physician or if the patient and family had already decided on palliative comfort measures, as per discussions with bedside nursing staff. Patients were excluded if the death was sudden or precipitous, occurring too quickly to initiate the $3 \mathrm{WP}$.

\section{Program implementation}

We introduced the program to the hospital ICU after a multidisciplinary reverse site visit to the originating institution,
St. Joseph's Healthcare Hamilton, followed by an on-site presentation at grand medical rounds. The original 3WP team facilitated a 1-day workshop that addressed the goals and genesis of the $3 \mathrm{WP}$, sharing examples of common wishes and suggested strategies for project initiation. Launch of the $3 \mathrm{WP}$ in the ICU at Brantford General Hospital was accomplished through the initiative of the local physician lead, support of other intensivists, the ICU Nurse Manager, the Spiritual Care team and a research coordinator. The local $3 \mathrm{WP}$ team comprised the nurse manager (B.L.), research coordinator (W.D.) and local lead investigator (B.R.).

A brief step-by-step guide to the $3 \mathrm{WP}$ program is provided in Appendix 1, available at www.cmajopen.ca/content/9/3/ E757/suppl/DC1. This tool guided clinical staff on the process and logistical steps to help organize the $3 \mathrm{WP}$ and integrate it into their care path. It shows the flow of activities for wish elicitation and facilitation. This guide was distributed during the initial multicentre evaluation, ${ }^{4}$ to provide direction on early conversations and introduce the $3 \mathrm{WP}$ to families.

Clinical staff and volunteers helped with data collection, communicated openly with families of dying patients, and created individualized keepsakes through direct discussions with patients and their loved ones. Various community members also participated, including a group who donated handknitted blankets. Once the program was clearly established, the hospital foundation offered ongoing support to purchase any needed supplies.

At semiannual retreats organized by the original project management office (B.R., W.D., D.J.C., F.C.), Brantford General staff and other local and international groups shared their data and experiences with each other; key activities included grand rounds (D.J.C., F.C., B.R.) on Sept. 20, 2017. Social media was instrumental in promoting this program, generating interest from local businesses, including a coffee shop that supplied a coffee maker with ongoing replenishment of coffees, teas and condiments. Families who used the program often donated supplies, such as books, toys, toiletries, tissues and rhythm strip vials (i.e., patient electrocardiogram strips inserted in glass vials as a keepsake for the family).

The costs of this 2-year program were covered primarily through donations, fundraising (W.D., B.R., B.L.) and grant support (D.J.C.). Grant funding was used exclusively for partial salary of the research coordinator, including 8 hours/week for the first 6 months and about 3 hours/week thereafter. Momentum was sustained by a successful in-hospital fundraiser (W.D., B.R., B.L.). Thereafter, occasional additional fundraising and numerous donations of consumables fuelled the clinical aspect of the program. We also created a periodic multidisciplinary staff newsletter about the 3WP (W.D., B.L.).

\section{Outcomes}

We selected outcomes in accordance with previous studies evaluating the $3 \mathrm{WP} .{ }^{4}$ Outcomes for this study included the type, timing and cost of each wish, which person or service made and facilitated each wish, and if and why the wish was successfully facilitated or not. Costs of wishes included direct costs of items purchased and consumables, such as materials 
for creating wishes, but did not include personnel time or other indirect costs, such as overhead. We also assessed patient enrolment and overall staff participation.

\section{Data collection}

The clinical team or research coordinator abstracted patient information from electronic and paper-based medical records onto pretested case report forms from the original $3 \mathrm{WP},{ }^{4}$ including demographics, admitting diagnosis, comorbidities, advanced life supports (including those administered, withheld and withdrawn), clinical course (i.e., length of stay in the ICU, time and location of death), clinician engagement, allied health services involved and family member presence at the time of death.

We calculated the Acute Physiology and Chronic Health Evaluation (APACHE II) score ${ }^{6}$ using biochemical values (e.g., creatinine, electrolytes, white blood cell count) and clinical data (e.g., vitals, Glasgow Coma Scale score, oxygen requirements) retrieved from the patient's medical chart. We did not abstract data in duplicate, given the clear nature of the data collected. No judgment was required. The research coordinator did, however, double-check the information recorded by bedside nurses, including information regarding implemented wishes and their cost. Although information was initially collected on paper for quick documentation (January 2017 to June 2017), this eventually moved to electronic medical records by bedside nurses, allowing for wishes to be recorded in real time. There was a 6-month interval between initiation of electronic record-keeping and paper documentation. The research coordinator collated the information from the case report forms and entered anonymized data using the encrypted software program REDCap.?

\section{Statistical analysis}

We performed all quantitative analyses using SAS Version 9.4. Descriptive statistics included means and standard deviations (SDs) for continuous variables, and absolute counts and percentages for categorical variables.

\section{Ethics approval}

This study was approved by the Brant Community Healthcare System Research Ethics Committee on Oct. 19, 2017. Approval for the original $3 \mathrm{WP}$ was under the Hamilton Integrated Research Ethics Board. ${ }^{3}$ This application was updated and approved in April 2017 to evaluate the $3 \mathrm{WP} .{ }^{4} \mathrm{We}$ used a waived consent model for quantitative data (i.e., basic characteristics and terminal wishes).

\section{Results}

We included 101 dying patients, with a mean age of 68.1 (SD 15.9) years; $56(55.4 \%)$ were female (Table 1). Patients were predominantly White $(n=95,94.1 \%) ; 5(5.0 \%)$ patients were Indigenous. Patients generally presented directly to the ICU from the emergency department; 91 (90.1\%) had medical admitting diagnoses, 7 (6.9\%) had surgical conditions and $3(3.0 \%)$ were admitted because of trauma. Life-support interventions were withdrawn immedi-
Table 1: Patient baseline demographic characteristics

\begin{tabular}{lc|} 
& No. (\%) of \\
Characteristic & patients \\
& $n=101$ \\
\hline
\end{tabular}

Age, yr, mean \pm SD

$68.1 \pm 15.9$

Sex, female

$56(55.4)$

Race

\begin{tabular}{l}
\hline White \\
\hline Indigenous \\
\hline Asian
\end{tabular}

\begin{tabular}{|l}
\hline APACHE II score, mean \pm SD \\
\hline Location before ICU \\
\hline Emergency department \\
\hline Hospital ward
\end{tabular}

Operating room

Other (inpatient rehabilitation ward)

$95(94.1)$

Other hospital (emergency department or ICU)

$5(5.0)$

$1(1.0)$

ICU admitting diagnosis

\begin{tabular}{lc} 
Cardiovascular or vascular & $20(19.8)$ \\
\hline Respiratory & $38(37.6)$ \\
\hline Gastrointestinal & $9(8.9)$ \\
\hline Neurologic & $10(9.9)$ \\
\hline Sepsis & $13(12.9)$ \\
\hline Other (e.g., trauma, metabolic) & $11(11.0)$
\end{tabular}

Admission category

Medical $91(90.1)$

Surgical $7(6.9)$

Trauma $3(3.0)$

Spiritual belief

Anglican $4(4.0)$

Baptist $8(7.9)$

Buddhist $1(1.0)$

Catholic $14(13.9)$

Christian $4(4.0)$

Eastern Orthodox $1(1.0)$

Jehovah's Witness $1(1.0)$

Longhouse $1(1.0)$

Lutheran $2(2.0)$

Pentecostal $1(1.0)$

Protestant 7 (6.9)

United 10 (9.9)

Unknown $14(13.9)$

None indicated $33(32.7)$

Note: ICU = intensive care unit, SD = standard deviation.

*Unless indicated otherwise.

ately before death for all patients, including mechanical ventilation from $60(59.4 \%)$ patients, and inotropes from $41(40.6 \%)$ patients (Table 2). Throughout the dying period, 


\begin{tabular}{|c|c|}
\hline Characteristic & $\begin{array}{c}\text { No. }(\%) \text { of patients* } \\
\qquad \begin{array}{l}n=101\end{array}\end{array}$ \\
\hline \multicolumn{2}{|l|}{ Advanced life supports at any time in ICU } \\
\hline Mechanical ventilation & $67(66.3)$ \\
\hline Inotropes & $65(64.4)$ \\
\hline Dialysis & $4(4.0)$ \\
\hline \multicolumn{2}{|l|}{ Advanced life supports withdrawn just before death } \\
\hline Mechanical ventilation & $60(59.4)$ \\
\hline Inotropes & $41(40.6)$ \\
\hline Dialysis & $0(0.0)$ \\
\hline \multicolumn{2}{|l|}{ Consults } \\
\hline Spiritual care & $38(37.6)$ \\
\hline Palliative care & $16(15.8)$ \\
\hline Social work & $53(52.5)$ \\
\hline Psychology & $1(1.0)$ \\
\hline Organ donation coordinator & $24(23.8)$ \\
\hline \multicolumn{2}{|l|}{ Consent for donation $†$} \\
\hline Yes, donation after cardiac death & $3(12.5)$ \\
\hline Yes, donation after neurologic death & $2(8.3)$ \\
\hline No donation made & $7(29.2)$ \\
\hline Patient ineligible for organ donation & $12(50.0)$ \\
\hline \multicolumn{2}{|l|}{ Patient death in hospital } \\
\hline Yes & $98(97.0)$ \\
\hline No & $2(2.0)$ \\
\hline Still in palliative unit & $1(1.0)$ \\
\hline \multicolumn{2}{|l|}{ Enrolment in 3WP initiated by } \\
\hline Principal investigator & $8(7.9)$ \\
\hline 3WP team & $5(5.0)$ \\
\hline Bedside nurse & $76(75.2)$ \\
\hline Spiritual care & $1(1.0)$ \\
\hline ICU attending physician & $4(4.0)$ \\
\hline Other & $7(6.9)$ \\
\hline Days from hospital admission to ICU admission, median (IQR) & $0(0-3)$ \\
\hline Days from ICU admission to death, median (IQR)‡ & $6(3-10)$ \\
\hline Days from hospital admission to death, median (IQR) & $9(4-16)$ \\
\hline Days from ICU admission to enrolment in 3WP, median (IQR) & $5(2-9)$ \\
\hline Days from enrolment in 3WP to death, median (IQR) $\ddagger$ & $1(0-1)$ \\
\hline \multicolumn{2}{|c|}{$\begin{array}{l}\text { Note: } 3 W P=3 \text { Wishes Project, ICU = intensive care unit, IQR = interquartile range. } \\
\text { *Unless indicated otherwise. } \\
\text { tOf } 24 \text { patients with a consult from organ donation coordinator. } \\
\text { fFor patients who did not die in hospital, the date of intensive care unit discharge was used to calculate days from hospital } \\
\text { admission to death, days from intensive care unit admission to death, and days from enrolment in } 3 \text { Wishes to death. }\end{array}$} \\
\hline
\end{tabular}

patients received consultations from spiritual care $(n=38$, $37.6 \%)$, palliative care $(n=16,15.8 \%)$, social work $(n=53$, $52.5 \%)$ and psychology $(n=1,1.0 \%)$.

The median length of stay in the ICU was 6 (interquartile range $[\mathrm{IQR}] 3-10)$ days. Enrolment in the $3 \mathrm{WP}$ was most often toward the end of ICU admission, with a median of 5 (IQR 2-9) days from ICU admission to enrolment in 3WP. Patients were introduced to the $3 \mathrm{WP}$ primarily by bedside nurses ( $n=76,75.2 \%$ ); the $3 \mathrm{WP}$ team (local lead investigator, research coordinator and nurse manager), other ICU physicians and the 
spiritual care team also introduced patients to the program. Of 101 patients, 98 (97.0\%) died in hospital, with 78 (79.6\%) dying in the ICU; a designated palliative care bed was secured for $11(11.2 \%)$ patients. At the time of death, $87(88.8 \%)$ patients had family or friends present at the bedside. Of 12 patients deemed eligible for organ donation, organ donation was realized for 5 patients, including 3 after cardiac death and 2 after neurologic death.

\section{Description of wishes}

Overall, a mean of 4.7 (SD 1.6) terminal wishes were facilitated for each patient (total range of 3-11 wishes/patient). The 479 total terminal wishes facilitated in this study represented a $99.2 \%$ completion rate; only 4 were not realized because of logistical or medical reasons (Table 3). Facilitated wishes represented each of the wish categories described in the original 3 WP study (Figure 1). Humanizing the environment included decorating a patient's room with memorabilia, such as emblems of the patient's birthplace region. Celebrations included a New Year's cheer, and arranging for an antique car show outside a patient's hospital window. Spiritual wishes included smudge ceremonies, a cedar bath, last rites, a wedding and bedside baptism. Keepsakes included paired crochet hearts, one to pin on a patient's chest and an identical heart as a family memento (Figure 2); another involved arranging a bedside ultrasound of a patient's partner to visualize their unborn child. Music included playing the patient's favourite songs on tablets or phones, and live performances with family and friends. For family connections, unlimited visiting hours and pet visits were arranged.

Most wishes were elicited while patients were still alive (97.7\%, 472 wishes), with only $2.3 \%$ (11 wishes) elicited in the postmortem period by families and friends. The ICU team elicited most wishes ( $n=363$ wishes, $75.2 \%)$, followed by family members $(n=103,21.3 \%)$ and the $3 \mathrm{WP}$ team $(n=17,3.5 \%)$. Wishes made directly by patients accounted for only $6.2 \%$ of wishes $(n=30)$, reflecting their critical illness; thus, family members and ICU staff wished for $238(48.9 \%)$ and $236(49.3 \%)$ wishes, respectively.

The mean cost per wish was $\$ 1.14$ (SD \$10.41); notably, $430(89.8 \%)$ of the 483 wishes elicited were at no cost to the program. The total cost of all 479 wishes facilitated for 101 patients was estimated to be $\$ 5.39$ per patient (SD \$22.40).

\section{Local program implementation}

Over the study period, all physicians and nurses, as well as other staff designated to the ICU (i.e., spiritual care clinicians, social workers, recreational therapists, pharmacists and dietitians) became aware of the $3 \mathrm{WP}$. We estimate that $70 \%-80 \%$ of the staff were personally involved in either eliciting or implementing a terminal wish. Staff reported that the step-bystep guide to implementing the $3 \mathrm{WP}$ was instrumental in its sustained uptake.

\section{Interpretation}

In this study, we documented successful implementation of the $3 \mathrm{WP}$ in the ICU of a community hospital. The program
Table 3: Summary of wishes from the 3 Wishes Project

\begin{tabular}{|c|c|}
\hline Characteristic & $\begin{array}{c}\text { No. }(\%) \text { of wishes } \\
n=483\end{array}$ \\
\hline \multicolumn{2}{|l|}{ Wish category } \\
\hline Family care & $60(12.4)$ \\
\hline Family connections & $50(10.4)$ \\
\hline Rituals and spiritual support & $50(10.4)$ \\
\hline Preparations and final arrangements & $41(8.5)$ \\
\hline Humanizing the patient & $26(5.4)$ \\
\hline Celebrations & $5(1.0)$ \\
\hline Humanizing the environment & $90(18.6)$ \\
\hline Keepsakes and tributes & $90(18.6)$ \\
\hline Music & $55(11.4)$ \\
\hline Word clouds & $12(2.5)$ \\
\hline Paying it forward & $2(0.4)$ \\
\hline Organ and tissue donation & $2(0.4)$ \\
\hline \multicolumn{2}{|l|}{ Who made the wish* } \\
\hline Patient & $30(6.2)$ \\
\hline Family & $236(48.9)$ \\
\hline Intensive care unit & $238(49.3)$ \\
\hline 3WP team & $0(0.0)$ \\
\hline $\begin{array}{l}\text { Other (friends, Trillium Gift of Life Network } \\
\text { staff, research staff) }\end{array}$ & $14(2.9)$ \\
\hline \multicolumn{2}{|l|}{ Wish made } \\
\hline Antemortem & $472(97.7)$ \\
\hline Postmortem & $11(2.3)$ \\
\hline \multicolumn{2}{|l|}{ Wish elicited by* } \\
\hline Patient & $7(1.4)$ \\
\hline Family & $103(21.3)$ \\
\hline Intensive care unit & $363(75.2)$ \\
\hline 3WP team & $17(3.5)$ \\
\hline $\begin{array}{l}\text { Other (chaplain, friends, Trillium Gift of Life } \\
\text { Network staff research staff) }\end{array}$ & $13(2.7)$ \\
\hline \multicolumn{2}{|l|}{ Wish completed } \\
\hline Yes & $479(99.2)$ \\
\hline No & $4(0.8)$ \\
\hline
\end{tabular}

helped to realize $99 \%$ of 483 terminal wishes in 101 dying, critically ill patients. Our findings illustrate how interprofessional clinicians can work synergistically to provide compassionate, individualized care for dying patients, including those who may have difficulty advocating for themselves.

On average, 5 terminal wishes per patient were facilitated. In this study, staff were more often involved than in the original $3 \mathrm{WP}$ report, ${ }^{4}$ accounting for close to half of the wishes made, in contrast to the $5 \%$ of wishes made by staff 


\section{cmaJOPEN}

Research

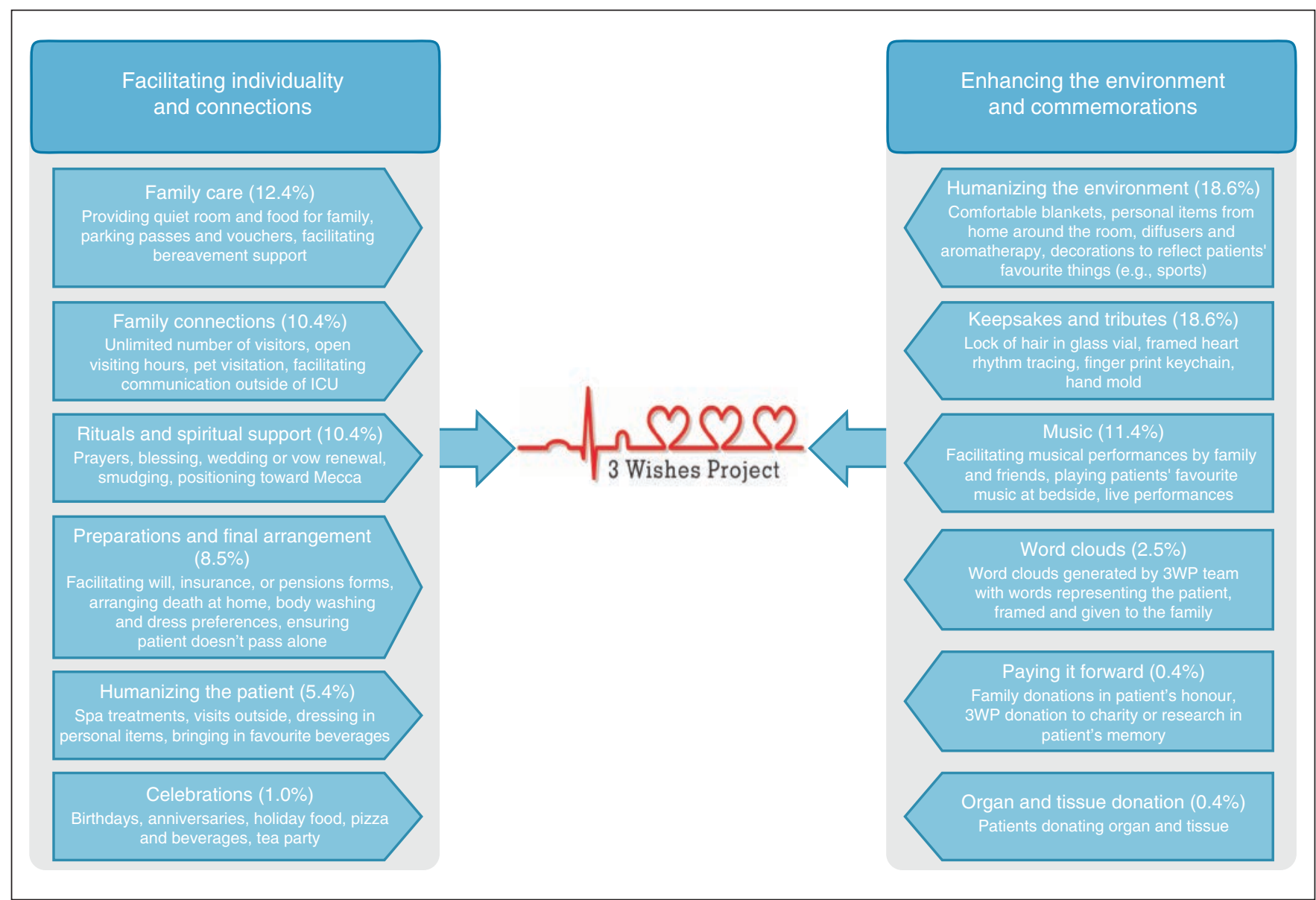

Figure 1: Visual pictograph outlining wish categories from the 3 Wishes Project (3WP), with a summary of examples. The percentages reported reflect the representation of wish categories among the 483 wishes documented during the study period. Note: ICU = intensive care unit. Categories were generated from a qualitative analysis during the original implementation of the 3 Wishes Project at St. Joseph's Healthcare Hamilton.

as reported in the original $3 \mathrm{WP}$ multisite program evaluation. ${ }^{4}$ Despite a similar proportion of about $90 \%$ of patients dying with family or close friends at the bedside in this community hospital and in the recent multicentre evaluation, ${ }^{4}$ the high proportion of wishes facilitated by ICU clinicians in this study reflects strong staff engagement.

Bedside nurses were not only responsible for enrolling $75 \%$ of patients, but they also facilitated $75 \%$ of the wishes, reflecting strong patient advocacy. Acknowledging that the provision of compassionate end-of-life care and facilitation of terminal wishes is not novel to nursing care, the formalization of this process through the $3 \mathrm{WP}$ helped to create norms, whereby compassionate acts are prevalent, approached with the means and structure to enable more consistent implementation. The $3 \mathrm{WP}$ emerged as a successful, nurse-championed hospital initiative, similar to rapid response critical care teams, ${ }^{8,9}$ smoking cessation clinics, ${ }^{10}$ diabetes education ${ }^{11}$ and hospital admission avoidance initiatives for older adults. ${ }^{12}$ Many nursing-led projects are cost-effective, ${ }^{11}$ are sustainable ${ }^{8}$ and favourably affect organizational culture, promoting effective communication between teams and patients. ${ }^{13}$

Although patients in this study appear to be less racially diverse than in the multicentre report, $(94.1 \% \mathrm{v}$.
$70.0 \%$ White), there was greater representation of Indigenous people $(5.0 \%$ v. $2.7 \%) .{ }^{4}$ The uptake of a palliative care program among First Nations communities is important, considering barriers to end-of-life care, such as isolation from families, limited access to public transportation, and cultural insensitivity to optimal end-of-life care for First Nations people. ${ }^{14}$ Trauma-informed care requires acknowledgement of the historic effects of colonialism, including organization-level discrimination and intergenerational trauma. ${ }^{14}$ It aims to prevent the perpetuation of discriminatory care stemming from Western misconceptions about First Nations culture. ${ }^{14,15} \mathrm{~W}$ ishes elicited from patients and families in this study aligned with trauma-informed care, fostering relationships with clinicians in the wish generation process. Moreover, the $3 \mathrm{WP}$ is a primarily patient- and family-led initiative, promoting the unique and culturally informed needs of all dying patients.

Beyond the favourable influence that the $3 \mathrm{WP}$ has for patients, family members and health care professionals, ${ }^{5}$ this study shows strong engagement of a community hospital in a combined clinical and research project. Community centres deliver most health care in Canada, accounting for $80 \%$ of the inpatient beds in Ontario, and 49\%-100\% in other provinces. ${ }^{16}$ 


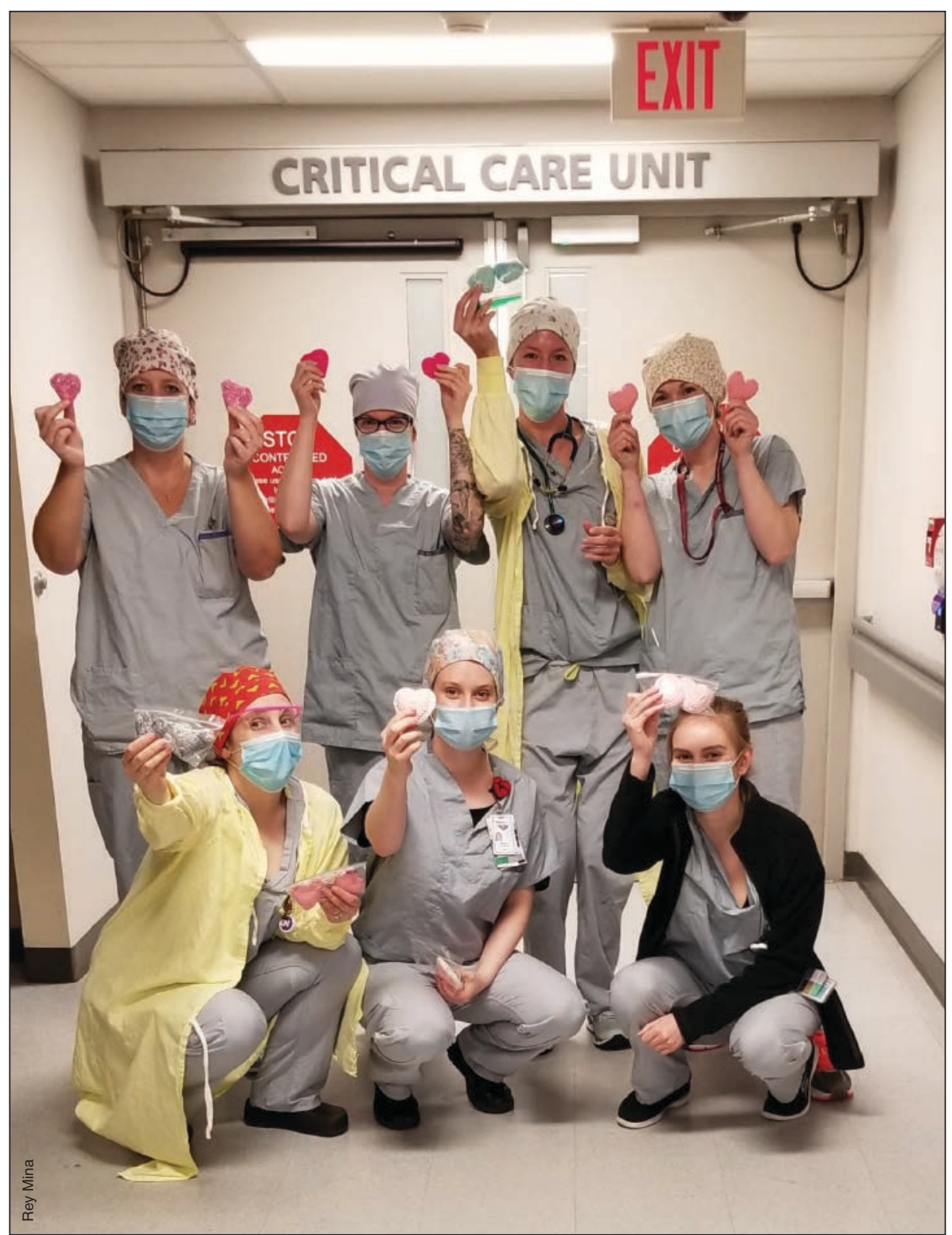

Figure 2: Community volunteers created pairs of crochet hearts, one to pin on a patient's chest and an identical heart for a family memento, as shown by bedside nurses. Top, left to right: Amy Warwick RN, Karyn Way RN, Danielle DeVries RN, Stephanie Ackland RN. Bottom, left to right: Alyssa Forler RN, Marin de Beer RN, Kara Jonas RN.

However, community centres are typically underrepresented in research generation, ${ }^{17}$ as most studies are designed and tested exclusively in academic settings. ${ }^{18}$ Previous work suggested that hospitals participating in research have improved outcomes for patients compared to hospitals that do not participate, ${ }^{18}$ including increased adherence to guidelines, ${ }^{19}$ higher nursing and physician satisfaction, and better employee retention..$^{20}$ Anticipating the barriers to research in the community setting, such as the delivery-focused model of care and their relatively fewer resources compared with academic centres, ${ }^{17}$ we documented successful uptake of the $3 \mathrm{WP}$, strong staff partnership and program sustainability beyond the research period. Our findings align with a perceived paradigm shift to increased research participation and academic contributions from community hospitals. . $^{17,18,21}$

Our work has shown the $3 \mathrm{WP}$ to function as a successfully and well-received, compassionate end-of-life program. Specifically, we showed that the program tapped into a desire for families to demonstrably honour the passing of loved ones, which may not have been realized before the invitation was given in the form of this program. The $3 \mathrm{WP}$ was a vehicle for enhanced recognition of each patient's inherent dignity, and a means to show compassion for patients and families while also individualizing care. Strengths include the realization of nearly all terminal wishes, achieving personalized, affordable, end-of-life interventions in this setting. Other differences 
from the original study ${ }^{3}$ and multicentre evaluation ${ }^{4}$ include nursing-led project implementation and early support from community agencies in our study.

\section{Limitations}

This study lacks qualitative interviews of patients, families and clinicians to draw on their experiences, which could have informed our understanding of the drivers of successful uptake of the $3 \mathrm{WP}$ and potential challenges to address. We did not perform duplicate data collection and entry for this study, so cannot exclude the possibility of mistakes, but these data are generally clear and not prone to error. We did not seek important measures of end-of-life quality in this study. Direct costs included only actual costs of wishes, not including costs of donated items.

\section{Conclusion}

The 3 Wishes Project is a patient- and family-centred palliative care initiative, successfully adapted to this community hospital at relatively low cost. The lack of strict protocolization and personalized design of this intervention underscores its inherent flexibility, with the potential to promote individualized end-oflife care in nonacademic hospital wards, homes or hospice. For consideration in other rural or remote venues, participatory research and more intentional cultural adaptation are needed.

\section{References}

1. Anderson I. Indigenous perspectives on death and dying. Toronto: University of Toronto; 2020.

2. Menec VH, Nowicki SKA. Transfers to acute care hospitals at the end of life: do rural/remote regions differ from urban regions? Rural Remote Health 2010;10:1281. Available: https://www.rrh.org.au/journal/article/1281 (accessed 2017 June 1)

3. Cook D, Swinton M, Toledo F, et al. Personalizing death in the intensive care unit: the 3 wishes project a mixed-methods study. Ann Intern Med 2015;163:271-9.

4. Vanstone M, Neville TH, Clarke FJ, et al. Compassionate end-of-life care: mixed-methods multisite evaluation of the 3 wishes project. Ann Intern Med 2020;172:1-11.

5. Neville TH, Agarwal N, Swinton M, et al. Improving end-of-life care in the intensive care unit: clinicians' experiences with the 3 Wishes Project. 7 Palliat Med 2019;22:1561-7.

6. Knaus WA, Draper EA, Wagner DP, et al. APACHE II: a severity of disease classification system. Crit Care Med 1985;13:818-29.

7. REDCap. Research Electronic Data Capture. Nashville: Vanderbilt University; 2004.

8. Mitchell A, Schatz M, Francis H. Designing a critical care nurse-led rapid response team using only available resources: 6 years later. Crit Care Nurse 2014;34:41-55; quiz 56 .

9. McNeill MM, Archer S, Remsburg D, et al. Rapid response team-quality champion registered nurse: observations and perceptions. 7 Nurs Care Qual 2019;34:325-9.

10. Byers MA, Wright P, Tilford JM, et al. Comparing smoking cessation outcomes in nurse-led and physician-led primary care visits. 7 Nurs Care Qual 2018;33:272-8.

11. Wilson M, Chen HS, Wood M. Impact of nurse champion on quality of care and outcomes in type 2 diabetes patients. Int 7 Evid-Based Healthc 2019;17:3-13.

12. Marsden E, Craswell A, Taylor A, et al. Nurse-led multidisciplinary initiatives to improve outcomes and reduce hospital admissions for older adults: The Care coordination through Emergency Department, Residential Aged Care and Primary Health Collaboration project. Australas f Ageing 2018;37:135-9.

13. Leach LS, Mayo AM. Rapid response teams: qualitative analysis of their effectiveness. Am 7 Crit Care 2013;22:198-210.

14. Russell B, Fred DE, Brown C. Culturally safe end-of-life care for First Nations persons living on reserve. Rural Remote Health 2018;18:4500. doi: 10.22605/ RRH4500.
15. Kelley ML, Prince H, Nadin S, et al. Developing palliative care programs in indigenous communities using participatory action research: a Canadian application of the public health approach to palliative care. Ann Palliat Med 2018;7(Suppl 2):S52-S72.

16. Number of hospital beds staffed and in operation, 2016-2017. Ottawa: Canadian Institute for Health Information; 2018. Available: www.cihi.ca/en/access -data-reports/results? query=Hospital+Beds+Staffed+and+In+Operation \% 2C+ 2016-2017\&Search+Submit $=($ accessed 2020 Sept. 14).

17. Gehrke P, Binnie A, Chan SPT, et al. Fostering community hospital research. CMA7 2019;191:E962-6.

18. DiDiodato G, Didiodato JA, McKee AS. The research activities of Ontario's large community acute care hospitals: a scoping review. BMC Health Serv Res 2017;17:566. doi: 10.1186/s12913-017-2517-4.

19. Luetz A, Balzer F, Radtke FM, et al. Delirium, sedation and analgesia in the intensive care unit: a multinational, two-part survey among intensivists. PLoS One 2014;9:e110935. doi: 10.1371/journal.pone.0110935.

20. Hacker K, Bhuiya N, Pernice J, et al. Assessing research interest and capacity in community health centers. Clin Transl Sci 2013;6:391-7.

21. Likumahuwa S, Song H, Singal R, et al. Building research infrastructure in community health centers: a Community Health Applied Research Network (CHARN) report. 7 Am Board Fam Med 2013;26:579-87.

Affiliations: Department of Critical Care (Reeve, Dechert, Longo, Scholes, Arthur), Brantford General Hospital, Brantford, Ont.; Department of Medicine (Dennis, Cook), McMaster University, Hamilton, Ont.; Department of Health Research Methods, Evaluation, and Impact (Dennis, Heels-Ansdell, Clarke, Cook), McMaster University, Hamilton, Ont.; Department of Critical Care (Dechert, Clarke, Cook), St. Joseph's Healthcare Hamilton, Ont.

Contributors: Brenda Reeve, William Dechert, Barbara Longo, France Clarke, John Arthur and Deborah Cook contributed to the conception and design of the work. William Dechert collected data, which all of the authors interpreted. Brenda Reeve and Brittany Dennis drafted the manuscript, and all authors revised it critically for important intellectual content, gave final approval of the version to be published and agreed to be accountable for all aspects of the work.

Funding: The research component was supported by the Canadian Institutes for Health Research (Foundation Grant 143243, Deborah Cook), which had no role in study design and conduct of the study, data collection, management, analysis, interpretation, or the preparation, review, or approval of the manuscript.

Content licence: This is an Open Access article distributed in accordance with the terms of the Creative Commons Attribution (CC BY-NC-ND 4.0) licence, which permits use, distribution and reproduction in any medium, provided that the original publication is properly cited, the use is noncommercial (i.e., research or educational use), and no modifications or adaptations are made. See: https://creativecommons.org/licenses/ by-nc-nd/4.0/

Data sharing: Summary data that support the findings of this study are available on request from the corresponding author. Individual patient data are not publicly available due to the identifying nature of some of the wishes.

Acknowledgements: The authors thank the patients and their loved ones for whom they had the honour of caring. The authors are grateful to the bedside nurses in the Critical Care Unit of the Brantford General Hospital, in addition to the physicians and other multidisciplinary colleagues who were instrumental in initiating and sustaining this program, including Ms. Tomoko Okomura (representing music therapy, Ms. Rachel Brown (representing recreation therapy), the Brant Community Healthcare System Foundation (led by Ms. Kari Wilson) and Mr. Tim Staffen, who manages the Tim Hortons. Additional leadership from ICU nurses, Ms. Laura Roth and Ms. Marin de Beer, and from Dr. Anna Rozenberg has been invaluable. The authors thank colleagues from the 3 Wishes Network for their assistance, especially the spiritual care clinician, Ms. Feli Toledo.

Supplemental information: For reviewer comments and the original submission of this manuscript, please see www.cmajopen.ca/content/9/3/ E757/suppl/DC1. 